\title{
Discovery and Follow-up Observations of the Young Type Ia Supernova 2016coj
}

\author{
WeiKang Zheng ${ }^{1}$, Alexei V. Filippenko ${ }^{1}$, Jon Mauerhan ${ }^{1}$, Melissa L. Graham ${ }^{1,2}$, Heechan Yuk $^{1}$, Griffin Hosseinzadeh ${ }^{3,4}$,
} Jeffrey M. Silverman ${ }^{5,18}$, Liming Rui ${ }^{6}$, Ron Arbour ${ }^{7}$, Ryan J. Foley ${ }^{8}$, Bela Abolfathi ${ }^{9}$, Louis E. Abramson ${ }^{10}$, Iair Arcavi ${ }^{3,4,19}$, Aaron J. Barth ${ }^{9}$, Vardha N. Bennert ${ }^{11}$, Andrew P. Brandel ${ }^{9}$, Michael C. Cooper ${ }^{9}$, Maren Cosens ${ }^{11}$, Sean P. Fillingham ${ }^{9}$, Benjamin J. Fulton ${ }^{12}$, Goni Halevi ${ }^{1}$, D. Andrew Howell ${ }^{3,4}$, Tiffany Hsyu ${ }^{8}$, Patrick L. Kelly ${ }^{1}$, Sahana Kumar ${ }^{1}$, Linyi Li ${ }^{6}$, Wenxiong $\mathrm{Li}^{6}$, Matthew A. Malkan ${ }^{10}$, Christina Manzano-King ${ }^{13}$, Curtis McCully ${ }^{3,4}$, Peter E. Nugent ${ }^{1,14}$, Yen-Chen Pan ${ }^{8}$, Liuyi Pei ${ }^{9}$, Bryan $\operatorname{Scott}^{13}$, Remington Oliver Sexton ${ }^{13}$, Isaac Shivvers ${ }^{1}$, Benjamin Stahl ${ }^{15}$, Tommaso Treu ${ }^{10}$, Stefano Valenti ${ }^{16}$,

\author{
H. Alexander Vogler ${ }^{9}$, Jonelle L. Walsh ${ }^{17}$, and Xiaofeng Wang ${ }^{6}$ \\ ${ }^{1}$ Department of Astronomy, University of California, Berkeley, CA 94720-3411, USA; zwk@astro.berkeley.edu \\ 2 Department of Astronomy, University of Washington, Box 351580, U.W., Seattle, WA 98195-1580, USA \\ ${ }^{3}$ Las Cumbres Observatory Global Telescope Network, 6740 Cortona Drive, Ste. 102, Goleta, CA 93117-5575, USA \\ ${ }^{4}$ Department of Physics, University of California, Santa Barbara, CA 93106-9530, USA \\ ${ }^{5}$ Department of Astronomy, University of Texas, Austin, TX 78712, USA \\ ${ }^{6}$ Physics Department and Tsinghua Center for Astrophysics, Tsinghua University, Beijing, 100084, China \\ ${ }_{7}^{7}$ Pennel Observatory, 29 Wrights Way, South Wonston, Hants S021 3He, UK \\ ${ }^{8}$ Department of Astronomy and Astrophysics, University of California, Santa Cruz, CA 95064, USA \\ ${ }^{9}$ Department of Physics and Astronomy, University of California, 4129 Frederick Reines Hall, Irvine, CA 92697, USA \\ ${ }^{0}$ Department of Physics and Astronomy, University of California, 430 Portola Plaza, Los Angeles, CA 90095, USA \\ ${ }_{11}^{11}$ Physics Department, California Polytechnic State University, San Luis Obispo, CA 93407, USA \\ ${ }^{12}$ Institute for Astronomy, University of Hawaii, 2680 Woodlawn Drive, Honolulu, HI 96822, USA \\ ${ }^{13}$ Department of Physics and Astronomy, University of California, 900 University Avenue, Riverside, CA 92521, USA \\ ${ }^{14}$ Lawrence Berkeley National Laboratory, Berkeley, California 94720, USA \\ ${ }^{15}$ Department of Physics, University of California, Berkeley, 94720, USA \\ ${ }^{16}$ Department of Physics, University of California, Davis, 1 Shields Avenue, Davis, CA 95616-5270, USA \\ ${ }^{17}$ George P. and Cynthia Woods Mitchell Institute for Fundamental Physics and Astronomy, Department of Physics and Astronomy, \\ Texas A\&M University, College Station, TX 77843, USA \\ Received 2016 November 28; revised 2017 April 17; accepted 2017 April 17; published 2017 May 24
}

\begin{abstract}
The Type Ia supernova (SN Ia) 2016coj in NGC 4125 (redshift $z=0.00452 \pm 0.00006$ ) was discovered by the Lick Observatory Supernova Search 4.9 days after the fitted first-light time (FFLT; 11.1 days before $B$-band maximum). Our first detection (prediscovery) is merely $0.6 \pm 0.5$ days after the FFLT, making SN 2016coj one of the earliest known detections of an SN Ia. A spectrum was taken only $3.7 \mathrm{hr}$ after discovery (5.0 days after the FFLT) and classified as a normal SN Ia. We performed high-quality photometry, low- and high-resolution spectroscopy, and spectropolarimetry, finding that SN 2016coj is a spectroscopically normal SN Ia, but the velocity of Si II $\lambda 6355$ around peak brightness $\left(\sim 12,600 \mathrm{~km} \mathbf{\nabla}^{1}\right)$ is a bit higher than that of typical normal SNe. The Si II $\lambda 6355$ velocity evolution can be well fit by a broken-power-law function for up to a month after the FFLT. SN 2016 coj has a normal peak luminosity $\left(M_{B} \approx-18.9 \mathbb{} t 0.2 \mathrm{mag}\right)$, and it reaches a $B$-band maximum $\sim 16.0$ days after the FFLT. We estimate there to be low host-galaxy extinction based on the absence of Na I D absorption lines in our low- and high-resolution spectra. The spectropolarimetric data exhibit weak polarization in the continuum, but the $\mathrm{Si}$ II line polarization is quite strong $(\sim 0.9 \% \pm 0.1 \%)$ at peak brightness.
\end{abstract}

Key words: supernovae: general - supernovae: individual (SN 2016coj)

Supporting material: data behind figure

\section{Introduction}

Type Ia supernovae (SNe Ia; see Filippenko 1997 for a review of supernova classification) are the thermonuclear runaway explosions of carbon/oxygen white dwarfs (see, e.g., Hillebrandt \& Niemeyer 2000 for a review). They can be used as standardizable candles with many important applications, including measurements of the expansion rate of the universe (Riess et al. 1998; Perlmutter et al. 1999). Two general scenarios are favored as the progenitor system for $\mathrm{SNe}$ Ia. One is the single-degenrate model (Hoyle \& Fowler 1960; Hachisu et al. 1996; Meng et al. 2009; Röpke et al. 2012), which consists of a single white dwarf accreting material from a companion. The other is the double-degenerate

\footnotetext{
${ }^{18}$ NSF Astronomy and Astrophysics Postdoctoral Fellow.

19 Einstein Fellow.
}

scenario involving the merger of two white dwarfs (Iben \& Tutukov 1984; Webbink 1984; Pakmor et al. 2012; Röpke et al. 2012). However, our understanding of their progenitor systems and explosion mechanisms remains substantially incomplete.

Very early discovery and detailed follow-up observations are essential for understanding those problems. For example, Bloom et al. (2012) were able to constrain the companionstar radius to be $\lesssim 0.1 \varangle R_{\odot}$ from an optical nondetection just $4 \mathrm{hr}$ after the explosion of SN 2011fe (Nugent et al. 2011). Cao et al. (2015) found strong but declining ultraviolet emission in SN Ia iPTF14atg in early-time Swift observations, consistent with theoretical expectations of the collision between supernova (SN) ejecta and a companion star (Kasen 2010). Im et al. (2015) found evidence of a "dark phase" in SN 2015F, which can last for a few hours to days between the moment of 
explosion and the first observed light (e.g., Rabinak et al. 2012; Piro \& Nakar 2013, 2014); see also Cao et al. (2016) for the case of iPTF14pdk. Differences in the duration of the "dark phase" could be caused by a varying distribution of ${ }^{56} \mathrm{Ni}$ near the surface of an SN Ia. For example, Piro \& Morozova (2016) show that it is short (with a steep rise) when the ${ }^{56} \mathrm{Ni}$ is shallow, and longer (with a more gradual rise) when the ${ }^{56} \mathrm{Ni}$ is deeper (see their Figure 7).

Spectra of SNe Ia not only reveal the ejecta composition from nuclear burning, but also provide a way to measure the ejecta expansion velocity. Benetti et al. (2005) separated SN Ia samples into different groups according to their velocity gradient and found that high-velocity-gradient objects tend to have a higher velocity of the Si II $\lambda 6355$ line near maximum light. Note that the higher velocity discussed in this paragraph is not the high-velocity feature described in Section 3.2. Nugent et al. (1995) quantified the spectral diversity using line-strength ratios, finding a good correlation between the absorption-depth ratio of $\mathrm{Si}$ II $\lambda 5972$ to $\mathrm{Si}$ II $\lambda 6355)$ and the brightness decline rate. Wang et al. (2009, 2013) and Foley \& Kasen (2011) separated SNe Ia into high-velocity and normal-velocity groups with a boundary at $11,800 \mathrm{~km} \mathrm{~s}^{-1}$ at peak brightness, and found that the former are $\sim 0.1$ mag (on average) redder in $B-V$ than the latter.

Meanwhile, high-resolution spectral observations provide a powerful way to study absorption along the line of sight, both from the interstellar medium and circumstellar material. Patat et al. (2007) found a complex of $\mathrm{Na}$ I D lines that showed evolution in SN 2006X (Wang et al. 2008). Two additional cases of time-variable $\mathrm{Na}$ absorption are provided by Blondin et al. (2009) and Simon et al. (2009). Sternberg et al. (2014) found that in their sample with high-resolution spectra, $\sim 18 \%$ of $\mathrm{SNe}$ Ia exhibit time-variable $\mathrm{Na}$, indicating the presence of circumstellar material and suggesting that it may be more common than expected in SNe Ia, though some objects do not show evolution (e.g., SN 2014J; Graham et al. 2015).

Spectropolarimetry can be used to probe the geometry of SNe Ia (see Wang \& Wheeler 2008 for a review). The continuum polarization, an indication of the photosphere's shape, was found to be quite low in $\mathrm{SNe} \mathrm{Ia}$, on the order of a few tenths of a percent (Höflich 1991; Wang et al. 1997). However, for individual SNe Ia, significant line polarization is sometimes observed (e.g., Kasen et al. 2003; Wang et al. 2003). Wang et al. (2007) also found a correlation between the degree of polarization of $\mathrm{Si}$ II $\lambda 6355$ and the brightness decline rate.

Observationally, there are numerous efforts to discover SNe Ia at very early times, which can benefit follow-up observations in many ways. Recent examples of early-observed and well-studied SNe Ia include SN 2009ig (Foley et al. 2012), SN 2011fe (Li et al. 2011; Nugent et al. 2011), SN 2012cg (Silverman et al. 2012b), SN 2013dy (Zheng et al. 2013), iPTF13ebh (Hsiao et al. 2015), SN 2014J (Goobar et al. 2014; Zheng et al. 2014; Graham et al. 2015), and ASASSN-14lp (Shappee et al. 2016); like SN 2016coj discussed here, they were either discovered or detected shortly after exploding.

In 2011, the observing strategy for our Lick Observatory Supernova Search (LOSS; Filippenko et al. 2001; Filippenko 2005; Leaman et al. 2011) with the 0.76 m Katzman Automatic Imaging Telescope (KAIT) was modified to monitor fewer galaxies but at a more rapid cadence, with the objective of promptly identifying very young $\mathrm{SNe}$ (hours to days after explosion). In the past few years, this strategy has led to discoveries of all types of young SNe, where we define an SN to be "young" if there was a KAIT nondetection one to three days before the first detection or if it was spectroscopically confirmed to be within a few days after explosion. SN 2012cg (Silverman et al. 2012b) was the first case, followed by more than a dozen others (e.g., SN 2012ck, Kandrashoff et al. 2012; SN 2012ea, Cenko et al. 2012; SN 2013ab, Blanchard et al. 2013; SN 2013dy, Zheng et al. 2013; SN 2013ej, Dhungana et al. 2016; SN 2013gh, Hayakawa et al. 2013; SN 2013fv, Kim et al. 2013a; SN 2013gd, Casper et al. 2013; SN 2013gy, Kim et al. 2013b; SN 2014C, Kim et al. 2014b; SN 2014J, though not discovered by KAIT, but with KAIT early detections, see Zheng et al. 2014; SN 2014ce, Kim et al. 2014a; SN 2014eh, Kumar et al. 2014; SN 2015N, Stegman et al. 2015a; SN 2015U, Shivvers et al. 2016; SN 2015X, Hughes et al. 2015; SN 2015O, Ross et al. 2015; SN 2015be, Stegman et al. 2015b; and SN 2016esw, discovered by Halevi et al. ${ }^{20}$ ).

SN 2016coj was another SN discovered by KAIT when very young, merely $0.6 \pm 0.5$ days after the fitted first-light time (FFLT). Here we present the first 40 days of our optical photometric, low- and high-resolution spectroscopic, and spectropolarimetric follow-up observations and analysis of it.

\section{Discovery and Observations}

SN 2016coj was discovered in an $18 \mathrm{~s}$ unfiltered KAIT image taken at 04:39:05 on 2016 May 28 (UT dates are used throughout this paper), at $14.98 \pm 0.03 \mathrm{mag}$ (close to the $R$ band; see Li et al. 2003). It was reported to the Transient Name Server (TNS) shortly after discovery by Yuk, Zheng, \& Filippenko $^{21}$ (see also Zheng et al. 2016). We measure its J2000.0 coordinates to be $\alpha=12^{\mathrm{h}} 08 \mathbf{Q}^{\circ} 6$ 6." $^{\prime \prime} 8, \delta=$ $+65 \bigotimes 10^{\prime} 38$." 2 , with an uncertainty of $0 .{ }^{\prime \prime} 5$ in each coordinate. SN 2016coj is 5!" 0 east and 10 $\$$ north of the nucleus of the host galaxy NGC 4125, which has redshift $z=0.00452$ $0.00006 \otimes($ de Vaucouleurs et al. 1991), an early-type peculiar elliptical morphology (E6 pec; de Vaucouleurs et al. 1991), and a stellar mass of $2.4 \varangle \times 10^{11 ष} M_{\odot}$ from its $3.6 \mu \mathrm{m}$ flux (Wilson et al. 2013).

KAIT performed photometric follow-up observations of SN 2016coj with nearly daily cadence after discovery. The data were reduced using our image-reduction pipeline (Ganeshalingam et al. 2010). We applied an image-subtraction procedure to remove host-galaxy light, and point-spreadfunction photometry was then obtained using DAOPHOT (Stetson 1987) from the IDL Astronomy User's Library. ${ }^{22}$ The unfiltered instrumental magnitudes, which are found to be close to the $R$ band ( $\mathrm{Li}$ et al. 2003), are calibrated to local SDSS standards (see Figure 1) transformed into Landolt $R$-band magnitudes. ${ }^{23}$ Here we publish our unfiltered photometry (Table 1). We have also obtained a filtered data sequence, but we are still awaiting high-quality galaxy template images in those bands.

Interestingly, we find that SN 2016coj was detected in a KAIT prediscovery image taken at 04:30:35 on May 24 (see middle panels of Figure 2) with an unfiltered mag of

\footnotetext{
${ }^{20}$ https://wis-tns.weizmann.ac.il//object/2016esw

21 https://wis-tns.weizmann.ac.il//object/2016coj

22 http://idlastro.gsfc.nasa.gov/

23 http://www.sdss.org/dr7/algorithms/sdssUBVRITransform. html\#Lupton2005
} 


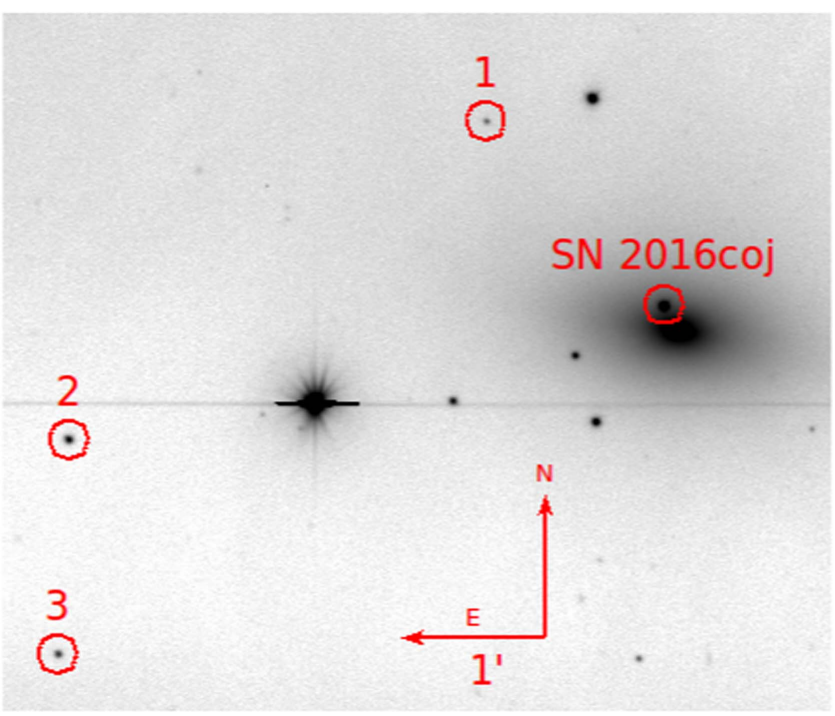

Figure 1. KAIT unfiltered image showing the location of SN 2016coj. Three reference stars are also marked with circles.

Table 1

Unfiltered Photometry of SN 2016coj

\begin{tabular}{|c|c|c|c|c|}
\hline MJD & UT & Mag & Error & From \\
\hline 57520.2370 & May 12.2370 & $>19.6$ & $\ldots$ & KAIT \\
\hline 57522.2513 & May 14.2513 & $>19.4$ & $\ldots$ & KAIT \\
\hline 57524.2240 & May 16.2240 & $>19.4$ & $\ldots$ & KAIT \\
\hline 57525.2479 & May 17.2479 & $>19.3$ & $\ldots$ & KAIT \\
\hline 57527.2708 & May 18.2708 & $>19.2$ & $\ldots$ & KAIT \\
\hline 57531.9092 & May 23.9092 & 18.06 & 0.42 & R. Arbour \\
\hline 57532.1877 & May 24.1877 & 18.02 & 0.22 & KAIT \\
\hline 57536.2694 & May 28.2694 & 14.98 & 0.03 & KAIT \\
\hline 57537.2196 & May 29.2196 & 14.50 & 0.04 & KAIT \\
\hline 57538.1827 & May 30.1827 & 14.11 & 0.03 & KAIT \\
\hline 57539.1814 & May 31.1814 & 13.85 & 0.03 & KAIT \\
\hline 57540.2518 & June 01.2518 & 13.54 & 0.04 & KAIT \\
\hline 57541.2022 & June 02.2022 & 13.39 & 0.06 & KAIT \\
\hline 57542.2075 & June 03.2075 & 13.18 & 0.03 & KAIT \\
\hline 57543.2120 & June 04.2120 & 13.16 & 0.03 & KAIT \\
\hline 57544.1995 & June 05.1995 & 13.02 & 0.03 & KAIT \\
\hline 57545.2245 & June 06.2245 & 13.00 & 0.03 & KAIT \\
\hline 57546.2200 & June 07.2200 & 12.95 & 0.03 & KAIT \\
\hline 57547.2051 & June 08.2051 & 12.93 & 0.03 & KAIT \\
\hline 57548.2197 & June 09.2197 & 12.94 & 0.04 & KAIT \\
\hline 57549.2162 & June 10.2162 & 12.99 & 0.05 & KAIT \\
\hline 57550.2638 & June 11.2638 & 12.97 & 0.02 & KAIT \\
\hline 57551.2201 & June 12.2201 & 13.01 & 0.03 & KAIT \\
\hline 57552.2516 & June 13.2516 & 13.03 & 0.03 & KAIT \\
\hline 57553.2115 & June 14.2115 & 13.15 & 0.03 & KAIT \\
\hline 57555.2189 & June 16.2189 & 13.27 & 0.03 & KAIT \\
\hline 57556.2284 & June 17.2284 & 13.30 & 0.03 & KAIT \\
\hline 57558.2110 & June 19.2110 & 13.52 & 0.02 & KAIT \\
\hline 57559.2229 & June 20.2229 & 13.61 & 0.04 & KAIT \\
\hline 57560.2189 & June 21.2189 & 13.66 & 0.04 & KAIT \\
\hline 57561.2097 & June 22.2097 & 13.70 & 0.04 & KAIT \\
\hline
\end{tabular}

$18.02 \pm 0.22$, which means the $\mathrm{SN}$ had brightened $\sim 3.0 \mathrm{mag}$ in the following four days until it was discovered. In addition, an unfiltered prediscovery detection was obtained at 21:48:57 on May 23, $6.7 \mathrm{hr}$ earlier than KAIT's first detection, by R. Arbour with a $0.35 \mathrm{~m} \mathrm{f} / 6$ Schmidt-Cassegrain reflector (see the left panels of Figure 2). Using a template image taken on 2016 April 5, we performed the same subtraction and

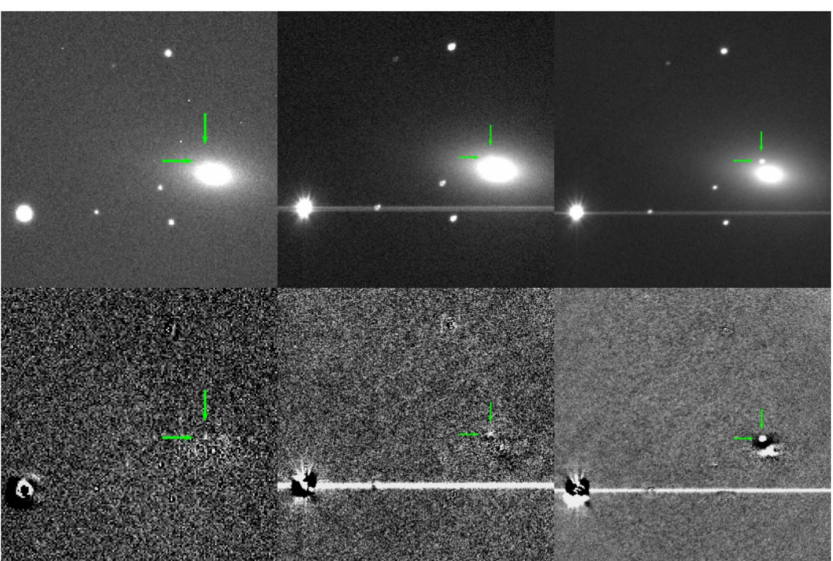

Figure 2. Left panels: Arbour's image taken on May 23. Middle panels: KAIT unfiltered image taken on May 24. Right panels: KAIT unfiltered image taken on May 28. Upper panels show the original image and lower panels show the residual after subtraction; SN 2016 coj is marked.

calibration methods as for the KAIT unfiltered images. We find an SN unfiltered brightness of $18.06 \pm 0.42 \mathrm{mag}$, consistent with KAIT's first detection. A $\sim 5$ mag detection before peak magnitude, along with our analysis in the following section, confirms that SN 2016coj is one of the youngest SNe Ia ever detected.

Two classification spectra of SN 2016coj were obtained shortly $(\sim 3.7 \mathrm{hr})$ after the $\mathrm{SN}$ was discovered ( $\sim 5.0$ days after the FFLT). The spectra were taken with the Kast double spectrograph (Miller \& Stone 1993) on the Shane 3 m telescope at Lick Observatory and the FLOYDS robotic spectrograph on the Las Cumbres Observatory Global Telescope Network (LCOGT; Brown et al. 2013) $2.0 \mathrm{~m}$ Faulkes Telescope North on Haleakala, Hawaii. We obtained nearly daily spectra of SN 2016coj with different instruments including Kast, FLOYDS, the BFOSC spectrograph on the $2.16 \mathrm{~m}$ telescope at Xinglong station of NAOC (China), the Low Resolution Imaging Spectrometer (LRIS; Oke et al. 1995) on the $10 \mathrm{~m}$ Keck I telescope, and the Kitt Peak Ohio State Multi-Object Spectrograph (KOSMOS; Martini et al. 2014) on the KPNO Mayall $4 \mathrm{~m}$ telescope. Data were reduced following standard techniques for $\mathrm{CCD}$ processing and spectrum extraction using IRAF. The spectra were flux calibrated through observations of appropriate spectrophotometric standard stars. All Kast and LRIS spectra were taken at or near the parallactic angle (Filippenko 1982) to minimize differential light losses caused by atmospheric dispersion. Low-order polynomial fits to calibration-lamp spectra were used to calibrate the wavelength scale, and small adjustments derived from night-sky lines in the target frames were applied. Flux calibration and telluric-band removal were done with our own IDL routines; details are described by Silverman et al. (2012a).

We also obtained four epochs of Lick/Shane spectropolarimetry using the polarimetry mode of the Kast spectrograph on May 30, June 8, June 16, and July 6. The spectra were observed at each of four waveplate angles $\left(0^{\circ}\right.$, $45^{\circ}, 22^{\circ} .5$, and $\left.67^{\circ} .5\right)$ with several waveplate sequences coadded to improve the signal-to-noise ratio $(\mathrm{S} / \mathrm{N})$. Each night, both low- and high-polarization standard stars were also observed in order to calibrate the data. All of the spectropolarimetric reductions and calculations follow the method described by Mauerhan et al. (2015). 




Figure 3. KAIT unfiltered (red) light curve of SN 2016coj. The solid black line is the $t^{2.0{ }^{2}}$ model fit to the red solid circles. The blue cross marks the earliest detection at $0.6 \pm 0.5$ days after the fitted first light observed by $\mathrm{R}$. Arbour. Red triangles show the KAIT upper limits before explosion.

In addition, we observed SN 2016coj on May 31, June 2, 4, and 6 with the $2.4 \mathrm{~m}$ Automated Planet Finder (APF) telescope at the Lick Observatory. The APF hosts the Levy Spectrograph, a high-resolution optical echelle spectrograph with resolution $R$ $(5500 \AA) \approx 110,000$ with a slit width of $1^{\prime \prime}$ (Vogt et al. 2014). At each epoch, we obtained three $1800 \mathrm{~s}$ spectra with the M decker (1"'0 wide, 8".0 long to allow for background subtraction), reduced the data with a custom pipeline, and corrected for the redshift of the host galaxy $(z=0.004523)$ and for the barycentric velocity (approximately $-15 \mathrm{~km} \mathrm{~s}^{-1}$ ). Because the apparent magnitude (peaking at $\sim 13 \mathrm{mag}$ ) of SN 2016coj is a bit faint for APF, the S/N of our spectra was $\lesssim 10$ đat best, significantly lower than that obtained with APF spectra of the bright (peak $\sim 10 \mathrm{mag}$ ), nearby SN Ia 2014J (Graham et al. 2015).

\section{Analysis and Results}

\subsection{Light Curve}

Figure 3 shows our unfiltered light curve of SN 2016coj. In order to determine the first-light time $t_{0}$ (note that the SN may exhibit a "dark phase"), one can assume that the SN luminosity scales as the surface area of the expanding fireball, and therefore increases quadratically with time $\left(L \propto t^{2}\right.$, commonly known as the $t^{2}$ model; Arnett 1982; Riess et al. 1999). The $t^{2}$ model fits well for several SNe Ia with early-time observations (e.g., SN 2011fe, Nugent et al. 2011 SN 2012ht, Yamanaka et al. 2014). Some studies also adopt a $t^{n}$ model ( $n$ varies from $\sim 1.5$ to $~ 3.0$; e.g., Conley et al. 2006; Ganeshalingam et al. 2011; Firth et al. 2015). Interestingly, Zheng et al. (2013, 2014) use a broken-power-law model to estimate the first-light time of SN 2013dy and SN 2014J. However, since our early-time photometric coverage of SN 2016coj is not as good as that of SN 2013dy and SN 2014J, we simply apply the $t^{2}$ model to fit the KAIT unfiltered data for the first few days (red solid circles in Figure 3); thereafter, the light curve starts deviating from the $t^{2}$ model. We also exclude Arbour's unfiltered detection (blue cross), considering the different response curve compared to the KAIT unfiltered data: Arbour's unfiltered band is closer to $V$ (see Botticella et al. 2009), while KAIT's is closer to $R$ (see Li et al. 2003).
We find that the best $t^{2}$ model fit gives the first-light time to be $\mathrm{MJD}=57531.33 \pm 0.50$, around May 23.33. Here the uncertainty (not including the "dark phase") is estimated by calculating the reduced $\chi^{2 \nabla_{\text {ratio }}}$ with the minimum reduced $\chi^{2 \otimes}$ at a $90 \%$ confidence level, when $t_{0}$ changes around the bestfitted value while all the other parameters are fixed with the best-fitted value. Note that the uncertainty does not include any systematic error caused by the $t^{2}$ model fitting. For example, if we include (or exclude) one data point before and after the data set we used, the best-fit first-light time deviates -0.4 to 1.0 days from the above first-light time. Therefore, there could be a systematic error of up to 1.0 day from this method, which we did not include in the following analysis. Our results show that the first detection (from an image by R. Arbour) was merely $0.6 \pm 0.5$ days after first light, or 0.9 days from KAIT's first detection on May 24. This makes SN 2016coj one of the earliest detected SNe Ia-slightly later than SN 2013dy $(\sim 2.4 \mathrm{hr}$ after first light; Zheng et al. 2013) and SN $2011 \mathrm{fe}$ ( $\sim 11.0 \mathrm{hr}$ after first light; Nugent et al. 2011), but similar to SN 2009ig ( $\sim 17 \mathrm{hr}$ after first light; Foley et al. 2012).

Applying a low-order polynomial fit, we find that SN 2016coj reached a peak magnitude of $12.91 \pm 0.03$ at MJD $=57547.31$ in KAIT unfiltered data. Although we do not present $B$-band data because no $B$-band template image is currently available, the fit allows us to determine the $B$-band peak time: $\mathrm{MJD}=57547.35$, similar to the result with unfiltered data. This means SN 2016coj was discovered only 4.9 days after the fitted first light, or 11.1 days before maximum light.

The distance modulus of the host galaxy NGC 4125 is quite uncertain (with a range of 30.04-32.80 mag) owing to different measurements (e.g., de Vaucouleurs \& Olson 1984; Tully 1988; Willick et al. 1997; Blakeslee et al. 2001; Tonry et al. 2001; Humphrey 2009; Tully et al. 2013). However, some of them are outdated, or adopted an inappropriate $H_{0}$ value. The one with the smallest uncertainty (and also the latest estimate) is $31.90 \pm 0.14 \mathrm{mag}$ (Tully et al. 2013), which was based on $H_{0 \rrbracket}=74.4 \llbracket \mathrm{km} \mathrm{s}^{-1} \mathrm{Mpc}^{-1}$, quite close to the current widely accepted value of $H_{0 \bigotimes} \approx 70 \rrbracket$ We therefore adopt this distance for the following ananlysis. With $E(B-V)_{\mathrm{MWD}}=0.02 \mathrm{mag}$ (Schlafly \& Finkbeiner 2011) and very small (even negligible) host-galaxy extinction (see Sections 3.2 and 3.5), this implies that SN 2016coj has $M_{R}=-19.0 \mathrm{Xt} 0.2 \mathrm{mag}$ at peak brightness. Our preliminary measurement of $B$-band data, assuming the host background contamination is small, shows that the $B$-band peak is $\sim 13.1 \pm 0.1 \mathrm{mag}$ and $\Delta m_{15}(B)=1.25 \varangle+0.12 \mathrm{mag}$. This gives $M_{B} \approx-18.9 \otimes t 0.2 \mathrm{mag}$, but we expect $M_{B} \approx-19.1$ hag from the Phillips (1993) relation with the above value of $\left.\Delta m_{15 \otimes} B\right)$; thus, SN 2016coj is a normal-brightness SN Ia. Its $\Delta m_{15}(B)=1.25 \nabla+0.12 \mathrm{mag}$ is also typical of normal SNe Ia (see also Section 3.3 for the spectral classification).

\subsection{Optical Spectra}

We obtained optical spectra of SN 2016coj nearly daily for a month (Figure 4), sometimes obtaining multiple spectra in a given night.

We first examine the Na I D absorption feature in several of our high-S/N spectra. The Na I D absorption feature is often converted into reddening, but with large scatter over the empirical relationship according to Poznanski et al. (2011); they estimated a systematic scatter of $0.3 \mathrm{mag}(1 \sigma)$ in $E(B-V)$ with the relation of the $\mathrm{Na}$ I $\mathrm{D}$ absorption equivalent 


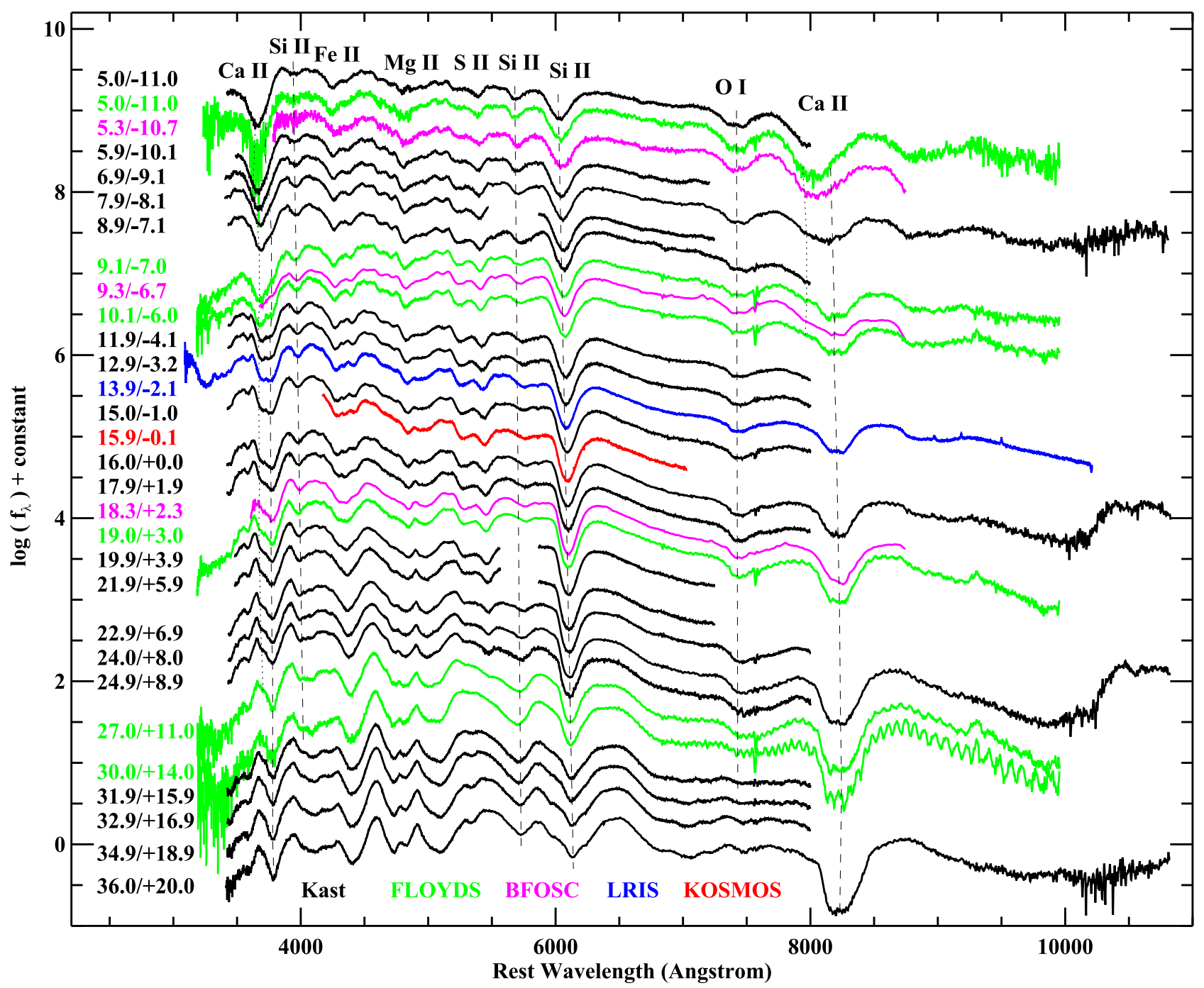

Figure 4. Spectral sequence of SN 2016coj over the first month after discovery. Each spectrum is labeled with its age relative to both the fitted first light and to the $B$-band maximum light. Some major spectral features are labeled at the top. Spectra taken by different instruments are shown in different colors. Three Lick/Kast spectra have no coverage around 5500-5900 ̊̊; those sections are left blank. Dashed lines are meant to help guide the eye when examining absorption features, while the dotted lines mark the high-velocity components of $\mathrm{Ca}$ II H\&K and the Ca II NIR triplet. The data used to create this figure are available.

width. The absorption is not clearly detected at the $\mathrm{Na}$ I D wavelength for both the Milky Way component and the hostgalaxy component. However, there appears to be a weak absorption feature consistent with the Milky Way Na I D wavelength. If real, this could be associated with the Milky Way extinction, which has $E(B-V)_{\mathrm{MW}}=0.02$ mag according to Schlafly \& Finkbeiner (2011). Since we do not detect similar absorption at the host-galaxy wavelength of $\mathrm{Na}$ I D, we can put an upper limit of $E(B-V) \lesssim 0.02 \mathrm{mag}$ on hostgalaxy extinction. However, if the weak absorption feature is caused by noise instead of Milky Way gas, we can determine an upper limit on $E(B-V)$ through comparison with our spectra of SN 2013dy (Zheng et al. 2013), where we clearly detect the $\mathrm{Na}$ I D absorption with the same instrument setting. For SN 2013dy, the equivalent width $\left(W_{\lambda}\right)$ is $\sim 0.5 \AA$ from both the Milky Way and host galaxy, giving $E(B-V)=0.15$ mag. Our similar-quality data on SN 2016coj should allow a detection of $1 / 3$ (or less) of Na I D absorption if it exists, yielding an upper limit of $E(B-V) \lesssim 0.05$ thag of host-galaxy extinction. Lastly, we also estimate a $3 \sigma$ upper limit on the $W_{\lambda}$ of an undetected feature in a spectrum using the equation presented by Leonard (2007): $W_{\lambda}(3 \otimes)=3 \varangle \lambda \Delta I \sqrt{W_{\text {lined }} \Delta \lambda} \sqrt{1 \not B}$, where $\Delta \lambda$ is the spectral resolution element (in $\AA$ ), $\Delta I$ is the $1 \sigma$ root-meansquare fluctuation of the flux around a normalized continuum level, $W_{\text {line® }}$ is the full-width at half-maximum intensity (FWHM) of the expected line feature, and $B$ is the number of bins per resolution element. For our high-S/N Kast spectra, we measure $\Delta \lambda \approx 4.0 \AA), \Delta \approx 0.015, W_{\text {line区 }} \approx 12.0 \varangle \AA$ \and $B=1$,

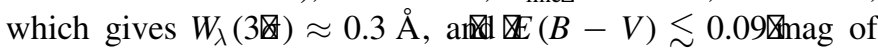
host-galaxy extinction.

All of the above suggests that the host-galaxy extinction of SN 2016coj is likely to be very small, consistent with the nondetection of NaI D absorption in our high-resolution spectra (see Section 3.5). However, note that since the Na I D versus extinction relation has large scatter, even a nondetection of $\mathrm{Na}$ I D does not fully exclude the possibility that there may be some dust along the SN line of sight.

The spectra show absorption features from ions typically seen in SNe Ia including Ca II, Si II, Fe II, Mg II, S II, and O I. We do not find a clear C II feature (e.g., Zheng et al. 2013), 


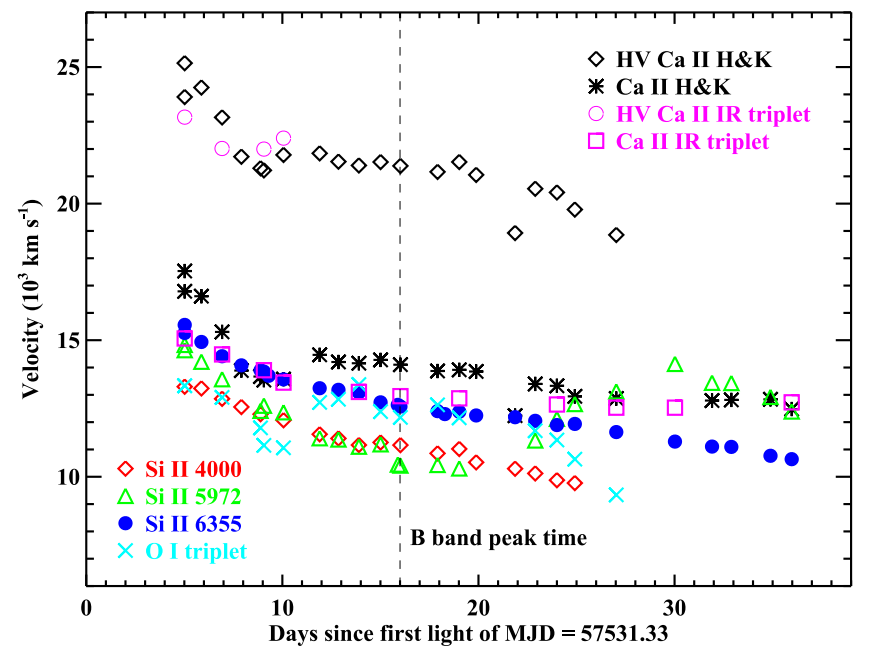

Figure 5. Expansion velocity evolution of different lines measured from the spectra of SN 2016coj. The black dashed line marks the time of $B$-band maximum light.

which is found in over one-fourth of all SNe Ia (e.g., Parrent et al. 2011; Thomas et al. 2011; Folatelli et al. 2012; Silverman \& Filippenko 2012). Strong absorption features of Si II, including Si II $\lambda 4000$, Si II $\lambda 5972$, and Si II $\lambda 6355$, are clearly seen in all spectra. The Si II $\lambda 5972$ feature in SN 2016coj is quite strong relative to those in SN 2012cg and SN 2013dy, though it is relatively small if compared with a large SN Ia sample (see Silverman et al. 2012c).

We measure the individual line velocities from the minimum of the absorption features (see Silverman et al. 2012c for details) and show them in Figure 5. The velocities of all Si II features decrease from $\sim 13,000-15,000 \mathrm{~km} \otimes \nabla^{1}$ at discovery to $\sim 11,000-13,000 \mathrm{~km} \nabla^{1}$ around maximum light, and they continue to decrease thereafter.

In addition to the usual photospheric-velocity feature (PVF) of Ca II H\&K, SN 2016coj exhibits a high-velocity feature (HVF; e.g., Mazzali et al. 2005; Maguire et al. 2012, 2014; Folatelli et al. 2013; Childress et al. 2014; Silverman et al. 2015) in nearly all of the early-time spectra. This HVF appears to be detached from the rest of the photosphere, with a velocity of $\sim 25,000 \mathrm{~km} \otimes \otimes^{1}$ at discovery and slowing down to $\sim 20,000 \mathrm{~km} \otimes 1$ at $\sim 8$ days after the fitted first-light time. The $\mathrm{HVF}$ feature of $\mathrm{Ca}$ II $\mathrm{H} \& \mathrm{~K}$ stays for a long time, being distinct until roughly age +11 days; thereafter, it is a high-velocity shoulder of the Ca II H\&K absorption.

A Ca II near-infrared (NIR) triplet HVF is also found in the first few spectra that covered the wavelength range before maximum light, and the velocity of $\gtrsim 22,000 \mathrm{~km} \$ \mathbb{Q}^{1}$ is in good agreement with that of the $\mathrm{Ca}$ II H\&K HVF at early times, though it is slightly smaller in the first-epoch spectrum. Such HVFs are seen in a few other well-observed SNe Ia, including SN 2005cf (Wang et al. 2009) and SN 2012fr (e.g., Childress et al. 2013; Maund et al. 2013; Zhang et al. 2014). However, in SN 2016coj, the Ca II NIR triplet HVF becomes weaker around peak brightness, and it completely disappears $\sim 8$ days later and thereafter. This is different from the Ca II H\&K HVF, which is seen for a much longer time. It is not obvious why the HVF of the Ca II NIR triplet goes away after peak brightness while the $\mathrm{HVF}$ of $\mathrm{Ca}$ II H\&K persists. One possibility is that the apparent HVF of Ca II H\&K after peak could actually be Si II $\lambda 3858$ (e.g., Foley 2013). In fact, it is possible that the early-time apparent HVF of Ca II H\&K could be a mixture of Si II $\lambda 3858$ (including both the HVF and PVF) plus the true HVF of Ca II $\mathrm{H} \& \mathrm{~K}$. If so, the velocity of the Ca II H\&K HVF could be smaller than that shown in Figure 5, and thus more consistent with the velocity of the Ca II NIR triplet HVF, but this case is too complicated to verify.

One note about the $\mathrm{O}$ I triplet feature is that we adopted only one component in our fit. However, our early-time spectra before peak brightness reveal that the $\mathrm{O}$ I triplet has a double absorption profile. Following the Zhao et al. (2016) method to fit the O I triplet with both HVF and PVH (Zhao et al. also adopted a second, faster HVF, but that is not clear in SN 2016coj), we find an HVF O I triplet velocity of $\sim 16,000 \mathrm{~km} \otimes \otimes^{1}$ and a PVF O I triplet velocity of $\sim 12,000 \mathrm{~km} \otimes \otimes 1$. The HVF velocity is smaller than that of both $\mathrm{Ca}$ II $\mathrm{H} \& \mathrm{~K}$ and the Ca II NIR triplet. If the HVF really exists in the $\mathrm{O}$ I triplet, it suggests that the oxygen in the outer layers is not completely burned (see Zhao et al. 2016).

The strong absorption of Si II $\lambda 6355$ is commonly used to estimate the photospheric velocity. As shown in Figure 5, the Si II $\lambda 6355$ velocity of SN 2016coj decreases rapidly from $\sim 15,500 \mathrm{~km} \otimes \otimes^{1}$ at discovery to $\sim 12,600 \mathrm{~km} \otimes \otimes^{1}$ around peak brightness, and then slowly decreases to $\sim 11,600 \mathrm{~km} \otimes \square^{1}$ at +11.0 days after peak. A velocity of $\sim 12,600 \mathrm{~km} \nabla^{1}$ at peak brightness is $\sim 1500 \mathrm{~km} \otimes \otimes^{1}$ higher than average in SNe Ia (e.g., Wang et al. $2013>2.5 \sigma$ away from the mean of their SN Ia velocity distribution fitted with a Gaussian).

Since the optical emission of SNe Ia comes mainly from the photosphere, the photospheric velocity evolution could be directly related to the optical light curves of SNe Ia (see Zheng \& Filippenko 2017); therefore, it is important to understand the photospheric velocity evolution of SNe Ia. Here, we compare the photospheric velocity measurement of SN 2016coj with the three well-observed SNe Ia 2009ig (Foley et al. 2012; Marion et al. 2013), 2012cg (Silverman et al. 2012b), and 2013dy (Zheng et al. 2013). Note that while both SN 2012cg and SN 2009ig have an HVF identified for Si II $\lambda 6355$, we consider only the photospheric component.

Figure 6 displays the photospheric velocity evolution over time for the four SNe Ia. Overall, the photospheric velocity evolution is similar to the evolution seen in most SNe Ia (e.g., Benetti et al. 2005; Foley et al. 2011; Silverman et al. 2012c): the velocity drops rapidly at early times (within the first week after explosion), and then slowly but steadily decreases thereafter. For each of these four $\mathrm{SNe}$, we consequently try to fit the early-time velocities (typically within 10 days after first light) with a power-law function, $v=C_{1 \mathbb{6}^{\prime}}{ }^{\alpha}$, where $t^{\prime}$ is the time after first light $\left(t_{0}\right)$; the results are shown in the top panel of Figure 6 for each SN. This is very similar to the method that of Silverman et al. (2015, Figure 12) adopted, but they used a natural exponential function to fit the velocities before +5 days after peak brightness and also obtained reasonable fitting results. In fact, Piro \& Nakar (2013, Equation (13)) mathematically show that the photospheric velocity could decay as a power law at early times. For the later velocities (typically $>10$ ddays after first light), we then fit them with a linear function, $v=a t^{\prime}+C_{2 \bigotimes}$ (results shown in the middle-top panel); Silverman et al. (2012c, Figure 5) also use the same method to fit their data around peak brightness. As seen in Figure 6, both the power-law function and the linear function can fit the corresponding data well, but only in their respective regimes - early-time data for the power-law function and latertime data for the linear function. 

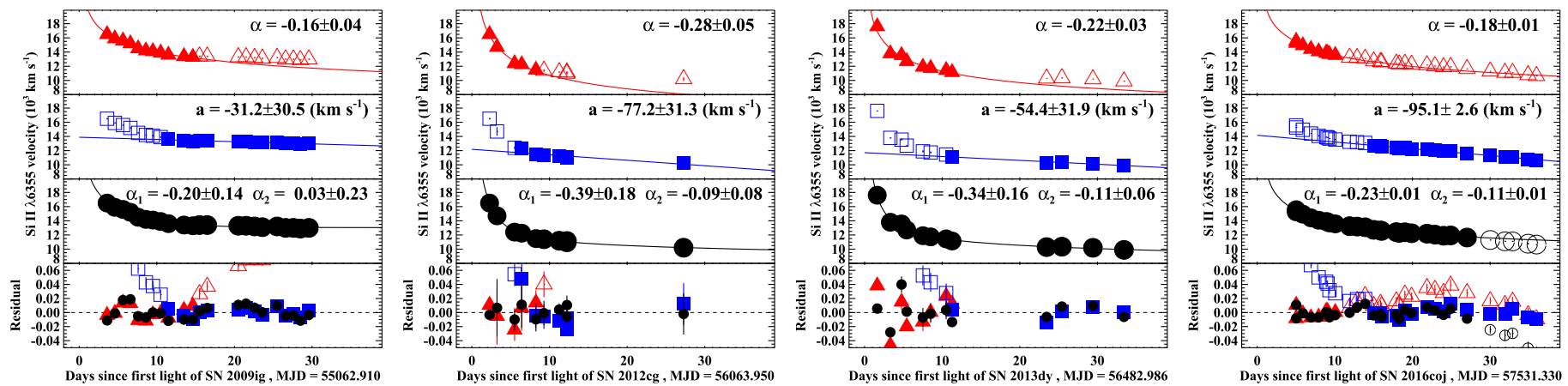

Figure 6. Photospheric velocity (measured from the strong Si II $\lambda 6355$ absorption) evolution of SN 2016coj (right panel), compared to those of the well-observed SN 2009ig (left panel), SN 2012cg (middle-left panel), and SN 2013dy (middle-right panel). For all SNe Ia, the top panels show the result of a power-law function fit to the early-time data, the middle-top panels display the result of a linear function fit to the later-time data, the middle-bottom panels give the result of a broken-powerlaw function fit to all the data, and the bottom panels show the residuals for each fit. Solid points are included in the fitting while open points are not.

Table 2

Photospheric Velocity Fitting Results

\begin{tabular}{lcccr}
\hline \hline & $\alpha$ & $a$ & $\alpha_{1}$ & $\alpha_{2}$ \\
\hline SN & Power Law & Linear $^{\mathrm{a}}$ & Broken Power Law $^{\mathrm{b}}$ \\
\hline SN 2009ig & $-0.16 \pm 0.04$ & $-32 \pm 31$ & $-0.20 \pm 0.14$ & $0.03 \pm 0.23$ \\
SN 2012cg & $-0.28 \pm 0.05$ & $-77 \pm 32$ & $-0.39 \pm 0.18$ & $-0.09 \pm 0.08$ \\
SN 2013dy & $-0.22 \pm 0.03$ & $-54 \pm 32$ & $-0.34 \pm 0.16$ & $-0.11 \pm 0.06$ \\
SN 2016coj & $-0.18 \pm 0.01$ & $-95 \pm 3$ & $-0.23 \pm 0.01$ & $-0.11 \pm 0.01$ \\
\hline
\end{tabular}

Notes.

${ }^{\mathrm{a}}$ In units of $\mathrm{km} \mathrm{s} \otimes \mathrm{day}^{-1}$.

${ }^{\mathrm{b}}$ The smoothing parameter $s$ was fixed to -10 during fitting, and the very small reduced $\chi^{2 \mathbb{8}_{\mathrm{f}}}$ some $\mathrm{SNe}$ is largely caused by overestimating the velocity uncertainty.

As with the early-time light curve (Zheng et al. 2013, 2014), we find that a broken-power-law function is useful for fitting the photospheric velocity evolution; a low-index power-law function approximates the linear function found at late times. Specifically,

$$
v=A\left(\frac{t^{\prime}}{t_{b}}\right)^{\alpha_{1}}\left[1+\left(\frac{t^{\prime}}{t_{b}}\right)^{s\left(\alpha_{1}-\alpha_{2}\right)}\right]^{-1 \rtimes_{s}}, \quad \otimes
$$

where $v$ is the photospheric velocity, $A$ is a scaling constant, $t^{\prime}$ is the time after first light $\left(t_{0}\right), t_{b}$ is the break time, $\alpha_{1}$ and $\alpha_{2}$ are the two power-law indices before and after the break (respectively), and $s$ is a smoothing parameter. We apply this broken-power-law function to the entire data set of photospheric velocities for all four SNe until about a month after the explosion. Our fitting results (we fixed $s$ to be -10 ) are listed in Table 2 and shown in the middle-bottom panels in Figure 6.

The power-law indices from both the power-law fitting $(\alpha)$ and broken-power-law fitting $\left(\alpha_{1}\right)$ at early times are consistent with the value of -0.22 adopted by Piro \& Nakar (2014) when fitting three SNe Ia (SNe 2009ig, 2011fe, and 2012cg), and are also the value adopted by Shappee et al. (2016) when fitting ASASSN-14lp. The index from the broken power law $\left(\alpha_{1}\right)$ is slightly steeper than that from the power law $(\alpha)$. At late times (around maximum light) with linear fitting, the rate of velocity decrease from the fitting is slightly larger than the average rate of $-38 \mathrm{~km} \mathrm{~s}^{-1}$ day $^{-1}$ found by Silverman et al. (2012c) for a large sample of SNe Ia.

Overall, the broken-power-law function can fit the photospheric velocity evolution well for all four SNe until a month after explosion (see the small residuals at the bottom panel of Figure 6 and the reduced $\chi^{2 区}$ given in Table 2). This function also has the potential to fit the photospheric velocity evolution of most other SNe Ia as well, given that most SNe Ia have very similar velocity evolution (e.g., Silverman \& Filippenko 2012; Silverman et al. 2012c). High-cadence spectroscopy is required to verify this, especially at early times. However, it currently remains unclear whether there is a good physical explanation behind the fitting; Piro \& Nakar (2013) show that the photospheric velocity could decay as a power law at early times, but our broken-power-law function fitting extends to a much later time.

\subsection{Classification}

We use the SuperNova IDentification code (SNID; Blondin \& Tonry 2007) to spectroscopically classify SN 2016coj. For nearly all of the spectra presented here, we find that SN 2016coj is spectroscopically similar to many normal SNe Ia. Compared to SN 1992A $\left(M_{B}=-18.79\right.$ mag and $\Delta m_{15 \mathrm{x}}(B)=1.47$ mag; Della Valle et al. 1998) and SN 2002er $\left(M_{B}=-19.35\right.$ mag and $\left.\Delta m_{15 \notin} B\right)=1.33 \mathrm{mag}$; Pignata et al. 2004), for example, SN 2016coj has similar spectra, absolute magnitude, and $\left.\Delta m_{15 \bigotimes} B\right)$. Another spectroscopic comparison is the so-called $\mathrm{Si}$ II ratio, $\mathfrak{R}$ (Si II) (the ratio of Si II $\lambda 5972$ to Si II $\lambda 6355)$, defined by Nugent et al. (1995) using the depths of spectral features and later by Hachinger et al. (2006) using their pseudo-equivalent widths. Hachinger et al. (2006) found a good correlation between the $\mathfrak{R}\left(\mathrm{Si}\right.$ II) and $\Delta m_{15 \bigotimes}(B)$ (see their Figure 13). We measure SN 2016coj to have $\mathfrak{R}(\mathrm{Si}$ II $)=0.11 \pm 0.4$, with $\Delta m_{15}(B)=$ 1.25 mag, placing SN 2016coj in the normal SN Ia region in Figure 13 of Hachinger et al. (2006), very close to SN 2002er. Thus, we conclude that SN 2016coj is a 
Table 3

Polarization of SN 2016coj

\begin{tabular}{lcccc}
\hline \hline Epoch & $P_{V}{ }^{\mathrm{a}}(\%)$ & $\theta_{V}(\mathrm{deg})$ & $P_{\text {cont } \boldsymbol{~}}$ & $\theta_{\text {cont }}(\mathrm{deg})$ \\
\hline 6.9 & $0.27(0.01)$ & $54.3(0.8)$ & $0.51(0.01)$ & $55.4(0.9)$ \\
16.0 & $0.20(0.01)$ & $52.6(0.8)$ & $0.38(0.01)$ & $53.4(0.8)$ \\
24.0 & $0.16(0.01)$ & $39.2(1.6)$ & $0.06(0.02)$ & $09.0(4.3)$ \\
44.0 & $0.39(0.04)$ & $41.0(1.3)$ & $0.28(0.03)$ & $30.0(2.2)$ \\
\hline
\end{tabular}

Note.

${ }^{\mathrm{a}} \mathrm{V}$-band and continuum integrated over wavelength ranges of 5050-5950 and 6700-7150 A, respectively. Uncertainties are statistical.

spectroscopically normal SN Ia, consistent with the photometric analysis given in Section 3.1.

\subsection{Spectropolarimetry}

Linear polarization is expressed as the quadratic sum of the $Q$ and $U$ Stokes vectors, $P=\sqrt{Q^{2 区}+U^{2 区}}$. The position angle on the sky is given by $\theta=(1 / 2)$ 政 $U / Q)$, taking into account the quadrant in the $Q-U$ plane where the inverse tangent angle is located. Since $P$ is a positive-definite quantity, it is overestimated in situations where the $\mathrm{S} / \mathrm{N}$ is low. It is thus typical to express the "debiased" (or bias-corrected) form of $P$ as $P_{\mathrm{db} \otimes}=\sqrt{\left(Q^{2}+U^{2 \otimes}\right)-\left(\sigma_{Q}^{2}+\sigma_{U}^{2 区}\right)}$, where $\sigma_{Q}$ and $\sigma_{U}$ are the uncertainties in the $Q$ and $U$ Stokes parameters. Note, however, that at low $\mathrm{S} / \mathrm{N}, P_{\mathrm{db} \otimes}$ is also not a fully reliable function because it has a peculiar probability distribution (Miller et al. 1988). Thus, for extracting statistically reliable values of polarization within a particular waveband, we have binned the calibrated $Q$ and $U$ Stokes spectra separately over the wavelength range of interest before calculating $P$ and $\theta$. All quoted and listed values in Table 3 were determined in this manner, while Figure 7 displays $P_{\mathrm{db}}$. For $\theta$, if $\left(\sigma_{Q}^{2}+\sigma_{U}^{2 \sqrt{3}}>\left(Q^{2}+U^{22}\right)\right.$, then we set a $1 \sigma$ upper limit on $P$ of $\sqrt{\sigma_{Q}^{2}+\sigma_{U}^{2 \otimes}}$. In cases where $P / \sigma_{P}<1.5 \rrbracket \theta$ is essentially undetermined and is not graphically displayed.

\subsubsection{Interstellar and Instrumental Polarization}

The interstellar polarization (ISP) appears to be low in the direction of SN 2016coj. Indeed, the estimated value of $E(B-V)=0.02 \mathrm{mag}$ indicates that the extinction from the Milky Way and host galaxy are not substantial; a small contribution from ISP is thus to be expected. According to Serkowski et al. (1975), an upper limit to the ISP is given by $9 \rrbracket \times E(B-V)$, which implies $P_{\mathrm{ISP} \otimes}<0.18 \%$ 区or SN 2016coj. To obtain a direct estimate of the Galactic component of ISP, we observed three Galactic stars in the vicinity of the SN position: HD 104436 (A3 V), HD 106998 (A5 V), and HD 108907 (M3 III). We measure respective $V$-band polarization and $\theta$ values of $P=(0.12 \%, 0.09 \%, 0.09 \%)$ and $\theta=\left(36^{\circ}\right.$, $34^{\circ}, 30^{\circ}$ ). Under the reasonable assumption of low intrinsic polarization for these stars, the resulting average values of $P \approx 0.1 \%, \theta \approx 33^{\circ}$ confirm the low Galactic polarization. Furthermore, the lack of Na I D absorption lines in our lowand high-resolution spectra (see Sections 3.2 and 3.5) indicates low extinction from the early-type host galaxy, and thus implies that the host ISP is probably even lower than the small Galactic value.

The instrumental polarization of the Kast instrument is also low. Measurements of the low-polarization standard star BD

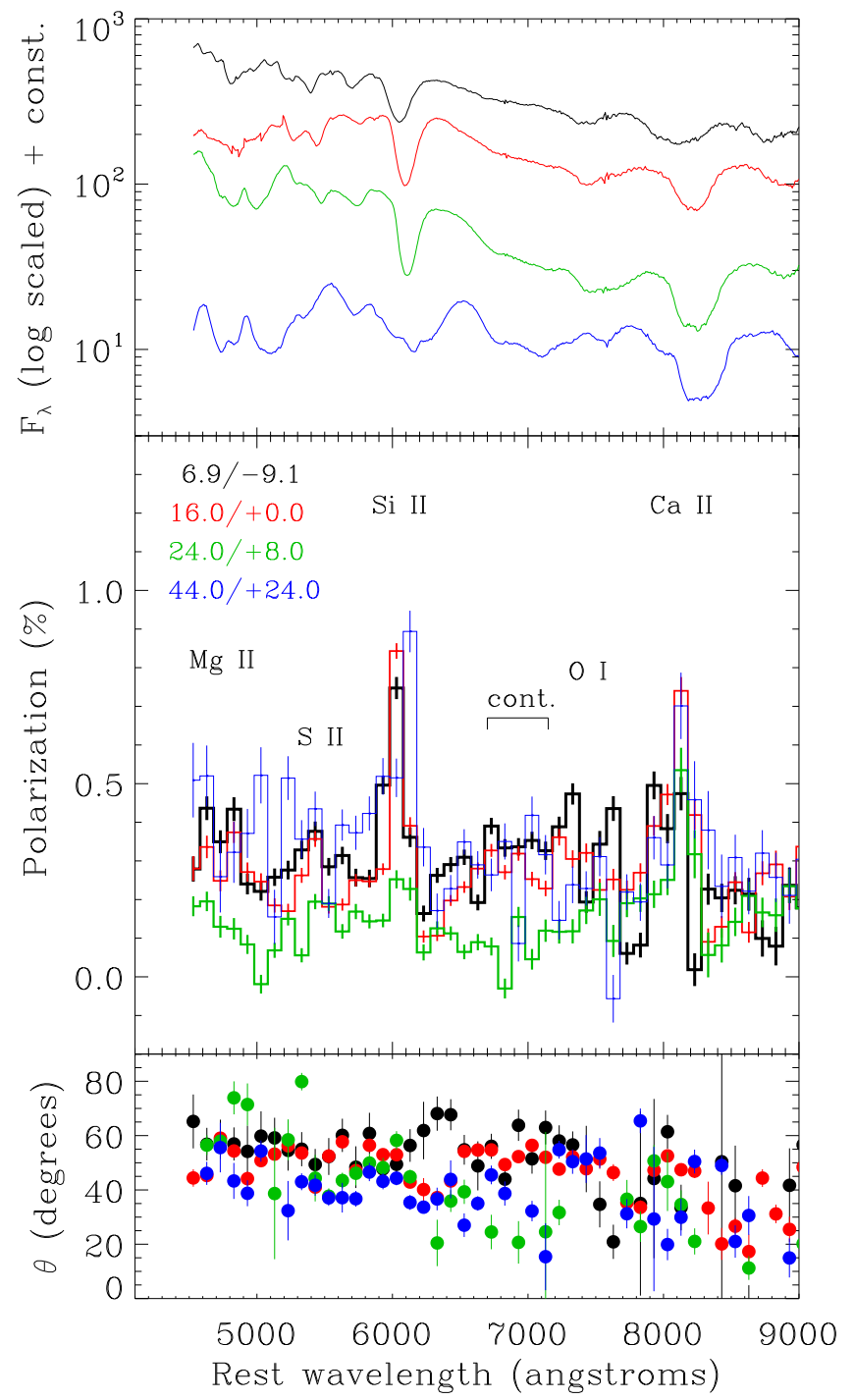

Figure 7. Four epochs of spectropolarimetry of SN 2016coj. Top panel: observed total-flux spectrum, color coded for each epoch. Middle panel: debiased polarization $\left(P_{\mathrm{db}}\right)$, with several major features labeled. Bottom panel: position angle $(\theta)$ for the corresponding epochs. The value of $\theta$ is undetermined where $P / \sigma_{P}<1.5$; $₫$ hose points are omitted.

+332642 at each epoch indicate an average $V$-band polarization of $\sim 0.15 \%$, with a standard deviation of $0.05 \%$ between all four epochs; the average value is consistent with that reported by Schmidt et al. (1992) for this star, which indicates that the low level is intrinsic to the source and that Kast contributes an insignificant amount of instrumental polarization to the measurements. The standard deviation is near the systematic uncertainty level we typically experience using the spectropolarimetry mode of Kast. Our observations therefore constrain the average instrumental polarization to $<0.05 \%$. Based on the low values of ISP and instrumental polarization, we move forward without attempting to subtract their minor contributions from the data.

\subsubsection{Intrinsic Polarization}

Our spectropolarimetry results are shown in Figure 7 and the integrated broadband measurements are listed in Table 3. On day 6.9 , the source exhibits weak polarization in the continuum at a level of $\sim 0.3 \%$, integrated over the wavelength range 
of $6700-7150 \AA$. This is consistent with the weak levels of continuum polarization that are typically associated with SNe Ia (Wang \& Wheeler 2008), though we note that some fraction of the polarization, perhaps half, could potentially be contributed by ISP. Strong polarization is exhibited across prominent line features, particularly Si II $\lambda 6355$ and the Ca II NIR triplet, at levels of $\sim 0.9 \%$ and $\sim 0.6 \%$, respectively. The $\mathrm{Ca}$ II polarization feature appears to exhibit two peaks, perhaps associated with the high- and low-velocity components. The position angles across the polarized line features, particularly $\mathrm{Si}$ II, are close to that of the continuum, which suggests an axisymmetric configuration for the $\mathrm{SN}$.

By day 16.0, the continuum polarization is consistent with having no change relative to day 6.9 , while Si II has increased in strength slightly to peak at this epoch. A Gaussian fit to the $\mathrm{Si}$ II feature indicates a line polarization of $0.9 \pm 0.1 \%$ with respect to the continuum level. For $\mathrm{Ca}$ II polarization, the enhancement of the high-velocity component from day 6.9 has disappeared and the peak of the lower-velocity component has increased by $\sim 0.3 \%$.

By day 24.0, the continuum and Si II line polarization appears to have dropped substantially for wavelengths shortward of $7000 \AA$, with no significant change apparent at longer wavelengths; polarization in the continuum region is undetected at this epoch. If real, such a continuum polarization drop roughly one week after peak would be reminiscent of the evolution of SN 2001el (Wang et al. 2003). However, by day 44.0 the continuum polarization appears to have regained the strength exhibited on day 16.0 and earlier. Si II has restrengthened as well, while declining in radial velocity along with the minimum of the weakening absorption profile. Based on this unexpected restrengthening, we exercise caution regarding the temporarily weakened polarization on day 24.0 because we are concerned that this could be the result of a systematic error. The drop in polarization appears to have only affected the Stokes $q$ parameter (derived from exposures with polarimeter waveplate angles at $0^{\circ}$ and $45^{\circ}$ ). Each of our three $q$ sequences of the SN are consistent, and we see no such change in the $q$ parameter of our standard-star observations from the same night. Thus, if the change on day 24.0 is the result of systematic error (e.g., some unknown temporary source of instrumental polarization above our typical limit of $<0.05 \%$ ), then it must have occurred over an hourly timescale. Alternatively, a subsequent rise in continuum polarization on day 44.0 could result from the appearance of weak line features in the chosen continuum region (6700-7150 $)$ ), but, in this case, we would not expect the simultaneous rise in the Si II feature. As a final possibility, the temporary influence of a separate light-echo component, possibly associated with dust in the host ISM, could result in the observed fluctuation; this possibility has the advantage of accounting for the brief change in the continuum and line polarization simultaneously, and it would also explain why the reddest wavelengths are not significantly affected.

Overall, the spectropolarimetric character of SN 2016coj is consistent with the trends exhibited by "normal" SNe Ia. For example, Maund et al. (2010) reported a correlation between the polarization of the Si II $\lambda 6355$ feature, measured near or before peak luminosity, and the radial-velocity decline rate of the absorption minimum (also see Leonard et al. 2006), physically interpreted as evidence for a single geometric configuration for normal SNe Ia. At peak brightness on day 16.0 , the line polarization of $0.9 \pm 0.1 \%$ combined with our measured value of $-95 \mathrm{~km} \mathrm{~s}^{-1}$ day $^{-1}$ for the velocity evolution, shows that SN 2016coj falls where expected on the correlated distribution of SNe Ia reported by Maund et al. (2010), and within the range of high-velocity explosions.

\subsection{High-resolution Spectra}

We examine the APF high-resolution spectra for narrow absorption features, such as those that were identified in APF spectra of SN 2014J (Graham et al. 2015). We began spectral monitoring with the APF based on an early classification and the assumption of a host-galaxy distance smaller than that adopted here. The object's peak apparent brightness ended up being $\sim 3$ mag fainter than that of SN 2014J, and fainter than the projected minimum we typically require for triggering the APF. For this reason, the S/N of our SN 2016coj APF spectra is quite low. Instead of ceasing our APF monitoring, we obtained multiple observations over several nights in order to stack our spectra, but ultimately we do not identify any absorption features of Na I D $\lambda \lambda 5889.95,5895.92$, Ca II H\&K $\lambda \lambda 3933.7,3968.5, \mathrm{~K}$ I $\lambda \lambda 7664.90,7698.96, \mathrm{H} \alpha \lambda 6562.801$, $\mathrm{H} \beta \lambda 4861.363$, or the diffuse interstellar bands $(\lambda \approx 5780$, 5797, 6196, 6283, $6613 \AA$ ).

Since the $\mathrm{Na}$ I D feature is most useful for constraining the presence of circumstellar material and line-of-sight host-galaxy dust extinction, and owing to grating blaze is in a region of relatively higher $\mathrm{S} / \mathrm{N}(\sim 10)$, we estimate an upper limit on its $W_{\lambda}$ in the following way. The flux of the continuumnormalized stacked APF spectra in the region of $\mathrm{NaI} D$, shown in black in Figure 8 , has a standard deviation of $\sigma \approx 0.038$. As an upper limit on the depth of an absorption feature that we could have detected, we use $3 \sigma \approx 0.11$. Our instrumental configuration for the Levy spectrograph results in a spectral resolution of $\Delta \lambda \approx 0.03 \AA$, from which we estimate that the minimum FWHM of a detected feature is $3 \Delta \lambda \approx 0.1 \AA$. Assuming a Gaussian profile for this hypothetical absorption, we constrain the $\mathrm{NaI} D$ feature to have $W_{\lambda} \lesssim 0.56 \varangle \AA$. Based on Figure 9 of Phillips et al. (2013), this puts an upper limit on host-galaxy extinction of $A_{V} \lesssim 0.2 \mathrm{mag}$ (with $E(B-V)=0.07$ mag assuming $R_{V}=3.1$ ). Although this is a rather large upper limit, it is consistent with the small host-galaxy extinction constrained from our low-resolution spectra (see Section 3.2) and also with the low extinction expected given the early type of the host, NGC 4125.

\section{Conclusions}

In this paper, we have presented optical photometric, lowand high-resolution spectroscopic, and spectropolarimetric observations of SN 2016coj, one of the youngest discovered and best-observed SNe Ia. Our clear-band light curve shows that our first detection is merely $0.6 \pm 0.5$ days after the FFLT, making it one of the earliest detected SNe Ia. We estimate that SN 2016coj took $\sim 16.0$ days after the fitted first-light time to reach $B$-band maximum. Its maximum brightness has a normal luminosity, $B=-18.9 \bigotimes \pm 0.2 \mathrm{mag}$. An estimated $\Delta m_{15 \bigotimes} B$ ) value of $1.25 \mathrm{mag}$ along with spectral information support its normal SN Ia classification. In the well-observed low-resolution spectral sequence, we identify a high-velocity feature from both $\mathrm{Ca}$ II $\mathrm{H} \& \mathrm{~K}$ and the $\mathrm{Ca}$ II NIR triplet, and also possibly from the O I triplet. SN 2016coj has a Si II $\lambda 6355$ velocity of $\sim 12,600 \mathrm{~km} \otimes \otimes^{1}$ at peak brightness, $\sim 1500 \mathrm{~km} \otimes \otimes^{1}$ higher than that of typical SNe Ia. We find that the Si II $\lambda 6355$ velocity 








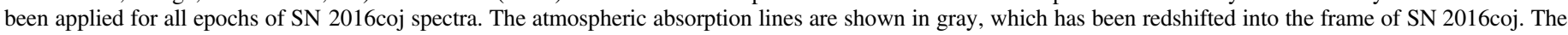

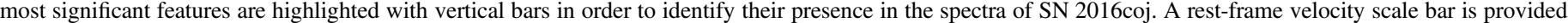
for each $\mathrm{Na} I \mathrm{D}$ feature along the top of the plot.

decreases rapidly during the first few days and then slowly decreases after peak brightness, very similar to that of other SNe Ia. A broken-power-law function can well fit the Si II $\lambda 6355$ velocity for up to about a month after first light. We estimate there to be very small host-galaxy extinction based on the lack of $\mathrm{Na}$ I D lines from the host galaxy in our low- and high-resolution spectra. Our four epochs of spectropolarimetry show that SN 2016coj exhibits weak polarization in the continuum, but the $\mathrm{Si}$ II line polarization is quite strong $(\sim 0.9 \% \pm 0.1 \%)$ at peak brightness.

Although SN 2016coj appears to be a normal SN Ia in many respects, it was detected very early (with the first detection merely $0.6 \pm 0.5$ days after the FFLT). So far, there are only a handful of SNe Ia that have been observed so soon after the explosion, making them very valuable at this stage to build up a bigger sample for studying $\mathrm{SNe}$ Ia at very early times. With new facilities coming online in the near future (e.g., the Zwicky Transient Facility; the Large Synoptic Survey Telescope), one can expect the number of such events to increase significantly, providing more opportunities for studying individual SNe Ia in greater detail shortly after explosion and also for performing statistical analyses.

A.V.F.'s group at U.C. Berkeley is grateful for financial assistance from National Science Foundation (NSF) grant
AST-1211916, Gary and Cynthia Bengier, the Richard and Rhoda Goldman Fund, the Christopher R. Redlich Fund, and the TABASGO Foundation. J.M.S. is supported by an NSF Astronomy and Astrophysics Postdoctoral Fellowship under award AST-1302771. UC Irvine observing runs were supported in part by NSF grant AST-1412693. Some of the data presented herein were obtained using the UC Berkeley and UC Irvine Remote Observing Facilities, made possible by generous gifts from John and Astrid Preston (UCB) and John and Ruth Ann Evans (UCI). V.N.B. and M.C. gratefully acknowledge assistance from an NSF Research at Undergraduate Institutions (RUI) grant AST-1312296. The UCSC group is supported in part by NSF grant AST-1518052, and by fellowships from the Alfred P. Sloan Foundation and the David and Lucile Packard Foundation to R.J.F. Observations by the UCLA group are supported by NSF grant AST-1412315 to T.T. The National Science Foundation of China financially supports X.W. (NSFC grants 11178003, 11325313, and 11633002). This work was partially supported by the Open Project Program of the Key Laboratory of Optical Astronomy, National Astronomical Observatories, Chinese Academy of Sciences. G.H., C.M., and D.A.H. are supported by NSF grant AST-1313484. Support for I.A. was provided by NASA through the Einstein Fellowship Program, grant PF6-170148. We thank the staffs of the various observatories at which data were obtained. Some of the data 
presented herein were obtained at the W. M. Keck Observatory, which was made possible by the generous financial support of the W. M. Keck Foundation. Based in part on observations obtained at Kitt Peak National Observatory, National Optical Astronomy Observatory (NOAO Prop. ID 2015B-0313; PI Foley), which is operated by the Association of Universities for Research in Astronomy (AURA), Inc., under cooperative agreement with the NSF. The authors are honored to be permitted to conduct astronomical research on Iolkam Du'ag (Kitt Peak), a mountain with particular significance to the Tohono O'odham. This work makes use of observations from the LCOGT network. We thank Lauren Weiss and Bradford Holden for obtaining APF data at Lick Observatory. KAIT and its ongoing operation were made possible by donations from Sun Microsystems, Inc., the Hewlett-Packard Company, AutoScope Corporation, Lick Observatory, the NSF, the University of California, the Sylvia and Jim Katzman Foundation, and the TABASGO Foundation. Research at the Lick Observatory is partially supported by a generous gift from Google.

\section{References}

Arnett, W. D. 1982, ApJ, 253, 785

Benetti, S., Cappellaro, E., Mazzali, P. A., et al. 2005, ApJ, 623, 1011 Blakeslee, J. P., Lucey, J. R., Barris, B. J., et al. 2001, MNRAS, 327, 1004 Blanchard, P., Zheng, W., Cenko, S. B., et al. 2013, CBET, 3422, 1 Blondin, S., Prieto, J. L., Patat, F., et al. 2009, ApJ, 693, 207 Blondin, S., \& Tonry, J. L. 2007, ApJ, 666, 1024 Bloom, J., Kasen, D., Shen, K., et al. 2012, ApJL, 744, L17 Botticella, M. T., Pastorello, A., Smartt, S. J., et al. 2009, MNRAS, 398, 1041 Brown, T. M., Baliber, N., Bianco, F. B., et al. 2013, PASP, 125, 1031 Cao, Y., Kulkarni, S. R., Gal-Yam, A., et al. 2016, ApJ, 832, 86 Cao, Y., Kulkarni, S. R., Howell, D. A., et al. 2015, Natur, 521, 328 Casper, C., Fuller, K. L., Zheng, W., et al. 2013, CBET, 3700, 1 Cenko, S. B., Li, W., Filippenko, A. V., et al. 2012, CBET, 3199, 1 Childress, M., Filippenko, A. V., Ganeshalingam, M., \& Schmidt, B. P. 2014, MNRAS, 437, 338

Childress, M., Scalzo, R. A., Sim, S. A., et al. 2013, ApJ, 770, 29

Conley, A., Howell, D. A., Howes, A., et al. 2006, ApJ, 132, 1707

de Vaucouleurs, G., de Vaucouleurs, A., Corwin, H. G., Jr., et al. 1991, RC3.9, Third Reference Catalogue of Bright Galaxies, version 3.9 (New York: Springer)

de Vaucouleurs, G., \& Olson, D. W. 1984, ApJS, 56, 91

Della Valle, M., Kissler-Patig, M., Danziger, J., \& Storm, J. 1998, MNRAS, 299, 267

Dhungana, G., Kehoe, R., Vinko, J., et al. 2016, ApJ, 822, 6

Filippenko, A. V. 1982, PASP, 94, 715

Filippenko, A. V. 1997, ARA\&A, 35, 309

Filippenko, A. V. 2005, in ASP Conf. Ser. 342, 1604-2004, Supernovae as Cosmological Lighthouses, ed. M. Turatto et al. (San Francisco, CA: ASP), 87

Filippenko, A. V., Li, W. D., Treffers, R. R., \& Modjaz, M. 2001, in ASP Conf. Ser. 246, Small-Telescope Astronomy on Global Scales, ed. B. Paczyński, W. P. Chen, \& C. Lemme (San Francisco, CA: ASP), 121

Firth, R. E., Sullivan, M., Gal-Yam, A., et al. 2015, MNRAS, 446, 3895

Folatelli, G., Morrell, N., Phillips, M. M., et al. 2013, ApJ, 773, 53

Folatelli, G., Phillips, M. M., Morrell, N., et al. 2012, ApJ, 745, 74

Foley, R. J. 2013, MNRAS, 435, 273

Foley, R. J., Challis, P. J., Filippenko, A. V., et al. 2012, ApJ, 744, 38

Foley, R. J., \& Kasen, D. 2011, ApJ, 729, 55

Foley, R. J., Sanders, N. E., \& Kirshner, R. P. 2011, ApJ, 742, 89

Ganeshalingam, M., Li, W., \& Filippenko, A. V. 2011, MNRAS, 416, 2607

Ganeshalingam, M., Li, W., Filippenko, A. V., et al. 2010, ApJS, 190, 418

Goobar, A., Johansson, J., Amanullah, R., et al. 2014, ApJL, 784, L12

Graham, M., Valenti, S., Fulton, B. J., et al. 2015, ApJ, 801, 136

Hachinger, S., Mazzali, P. A., \& Benetti, S. 2006, MNRAS, 370, 299

Hachisu, I., Kato, M., \& Nomoto, K. 1996, ApJL, 470, L97

Hayakawa, K., Cenko, S. B., Zheng, W., et al. 2013, CBET, 3706, 1

Hillebrandt, W., \& Niemeyer, J. C. 2000, ARA\&A, 38, 191

Höflich, P. 1991, A\&A, 246, 481
Hoyle, F., \& Fowler, W. A. 1960, ApJ, 132, 565

Hsiao, E. Y., Burns, C. R., Contreras, C., et al. 2015, A\&A, 578, 9

Hughes, A., Zheng, W., \& Filippenko, A. V. 2015, CBET, 4169, 1 Humphrey, P. J. 2009, ApJ, 690, 512

Iben, I., Jr., \& Tutukov, A. V. 1984, ApJS, 54, 335

Im, M., Choi, C., Yoon, S., et al. 2015, ApJS, 221, 22

Kandrashoff, M., Fuller, K., Cenko, S. B., et al. 2012, CBET, 3121, 1

Kasen, D. 2010, ApJ, 708, 1025

Kasen, D., Nugent, P. E., Wang, L., et al. 2003, ApJ, 593, 788

Kim, H., Casper, C., Zheng, W., et al. 2013a, CBET, 3678, 1

Kim, H., Zheng, W., Filippenko, A. V., et al. 2014a, CBET, 3942, 1

Kim, H., Zheng, W., Li, W., et al. 2013b, CBET, 3743, 1

Kim, M., Zheng, W., Li, W., \& Filippenko, A. V. 2014b, CBET, 3777, 1

Kumar, S., Yuk, H., Zheng, W., et al. 2014, CBET, 4165, 1

Leaman, J., Li, W., Chornock, R., \& Filippenko, A. V. 2011, MNRAS, 412,1419

Leonard, D. 2007, ApJ, 670, 1275

Leonard, D. C., Filippenko, A. V., Ganeshalingam, M., et al. 2006, Natur, 440,505

Li, W., Bloom, J. S., Podsiadlowski, P., et al. 2011, Natur, 480, 348

Li, W., Filippenko, A. V., Chornock, R., \& Jha, S. 2003, PASP, 115, 844

Maguire, K., Sullivan, M., Ellis, R. S., et al. 2012, MNRAS, 426, 2359

Maguire, K., Sullivan, M., Pan, Y., et al. 2014, MNRAS, 444, 3258

Marion, G. H., Vinko, J., Wheeler, J. C., et al. 2013, ApJ, 777, 40

Martini, P., Elias, J., Points, S., et al. 2014, Proc. SPIE, 9147, 91470Z

Mauerhan, J. C., Williams, G. G., Leonard, D., et al. 2015, MNRAS, 453, 4467

Maund, J. R., Höflich, P., Patat, F., et al. 2010, ApJL, 725, L167

Maund, J. R., Spyromilio, J., Höflich, P. A., et al. 2013, MNRAS, 433, L20

Mazzali, P. A., Benetti, S., Altavilla, G., et al. 2005, ApJL, 623, L37

Meng, X., Chen, X., Han, Z., et al. 2009, MNRAS, 395, 2103

Miller, J. S., Robinson, L. B., \& Goodrich, R. W. 1988, in Instrumentation for Ground-Based Optical Astronomy, ed. L. B. Robinson (New York: Springer), 157

Miller, J. S., \& Stone, R. P. S. 1993, Lick Obs. Tech. Rep. 66 (Santa Cruz: Lick Obs)

Nugent, P. E., Phillips, M. M., Baron, E., Branch, D., \& Hauschildt, P. 1995, ApJL, 455, L147

Nugent, P. E., Sullivan, M., Cenko, S. B., et al. 2011, Natur, 480, 344

Oke, J. B., Cohen, J. G., Carr, M., et al. 1995, PASP, 107, 375

Pakmor, R., Kromer, M., Taubenberger, S., et al. 2012, ApJL, 747, L10

Parrent, J. T., Thomas, R. C., Fesen, R. A., et al. 2011, ApJ, 732, 30

Patat, F., Chandra, P., Chevalier, R., et al. 2007, Sci, 317, 924

Perlmutter, S., Aldering, G., Goldhaber, G., et al. 1999, ApJ, 517, 565

Phillips, M. M. 1993, ApJL, 413, L105

Phillips, M. M., Simon, J. D., Morrell, N., et al. 2013, ApJ, 779, 38

Pignata, G., Patat, F., Benetti, S., et al. 2004, MNRAS, 355, 178

Piro, A. L., \& Morozova, V. S. 2016, ApJ, 826, 96

Piro, A. L., \& Nakar, E. 2013, ApJ, 769, 67

Piro, A. L., \& Nakar, E. 2014, ApJ, 784, 85

Poznanski, D., Ganeshalingam, M., Silverman, J. M., \& Filippenko, A. V. 2011, MNRAS, 415, L81

Rabinak, I., Livne, E., \& Waxman, E. 2012, ApJ, 757, 35

Riess, A. G., Filippenko, A. V., Challis, P., et al. 1998, AJ, 116, 1009

Riess, A. G., Filippenko, A. V., Li, W., et al. 1999, AJ, 118, 2675

Röpke, F. K., Kromer, M., Seitenzahl, I. R., et al. 2012, ApJL, 750, L19

Ross, T. W., Zheng, W., Filippenko, A. V., et al. 2015, CBET, 4125, 1

Schlafly, E. F., \& Finkbeiner, D. P. 2011, ApJ, 737, 103

Schmidt, G. D., Elston, R., \& Lupie, O. L. 1992, AJ, 104, 1563

Serkowski, K., Mathewson, D. S., \& Ford, V. L. 1975, ApJ, 196, 261

Shappee, B. J., Piro, A. L., Holoien, T. W., et al. 2016, ApJ, 826, 144

Shivvers, I., Zheng, W., Mauerhan, J., et al. 2016, MNRAS, 461, 3057

Silverman, J. M., \& Filippenko, A. V. 2012, MNRAS, 425, 1917

Silverman, J. M., Foley, R., Filippenko, A. V., et al. 2012a, MNRAS, 425,1789

Silverman, J. M., Ganeshalingam, M., Cenko, S. B., et al. 2012b, ApJL, 756, L7

Silverman, J. M., Kong, J. J., \& Filippenko, A. V. 2012c, MNRAS, 425, 1819 Silverman, J. M., Vinkó, J., Marion, G. H., et al. 2015, MNRAS, 451, 1973

Simon, J. D., Gal-Yam, A., Gnat, O., et al. 2009, ApJ, 702, 1157

Stegman, S., Zheng, W., \& Filippenko, A. V. 2015a, CBET, 4124, 1

Stegman, S., Zheng, W., \& Filippenko, A. V. 2015b, CBET, 4128, 1

Sternberg, A., Gal-Yam, A., Simon, J. D., et al. 2014, MNRAS, 443, 1849

Stetson, P. B. 1987, PASP, 99, 191

Thomas, R. C., Aldering, G., Antilogus, P., et al. 2011, ApJ, 743, 27

Tonry, J. L., Dressler, A., Blakeslee, J. P., et al. 2001, ApJ, 546, 681

Tully, R. B. 1988, Nearby Galaxy Catalog (Cambridge: Cambridge Univ. Press) 
Tully, R. B., Courtois, H. M., Dolphin, A. E., et al. 2013, AJ, 146, 86

Vogt, S., Radovan, M., Kibrick, R., et al. 2014, PASP, 126, 359

Wang, L., Baade, D., Höflich, P., et al. 2003, ApJ, 591, 1110

Wang, L., Baade, D., \& Patat, F. 2007, Sci, 315, 212

Wang, L., \& Wheeler, J. C. 2008, ARA\&A, 46, 433

Wang, L., Wheeler, J. C., \& Höflich, P. 1997, ApJL, 476, L27

Wang, X., Li, W., Filippenko, A. V., et al. 2008, ApJ, 675, 626

Wang, X., Li, W., Filippenko, A. V., et al. 2009, ApJ, 697, 380

Wang, X., Wang, L., Filippenko, A. V., Zhang, T., \& Zhao, X. 2013, Sci, 340,170
Webbink, R. F. 1984, ApJ, 277, 355

Willick, J. A., Courteau, S., Faber, S. M., et al. 1997, ApJS, 109, 333

Wilson, C. D., Cridland, A., Foyle, K., et al. 2013, ApJ, 776, 30

Yamanaka, M., Maeda, K., Kawabata, M., et al. 2014, ApJL, 782, L35

Zhang, J., Wang, X., Bai, J., et al. 2014, AJ, 148, 1

Zhao, X. L., Maeda, K., Wang, X., et al. 2016, ApJ, 826, 211

Zheng, W., \& Filippenko, A. V. 2017, ApJL, 838, L4

Zheng, W., Shivvers, I., Filippenko, A. V., et al. 2014, ApJL, 783, L24

Zheng, W., Silverman, J. M., Filippenko, A. V., et al. 2013, ApJL, 778, L15

Zheng, W., Yuk, H., Manzano-King, C., et al. 2016, ATel, 9095, 1 